\title{
A 10-year cohort study of the burden and risk of in-hospital falls and fractures using routinely collected hospital data
}

\author{
C A Brand, ${ }^{1} \vee$ Sundararajan ${ }^{2}$
}

${ }^{1}$ Centre of Research Excellence in Patient Safety (CREPS), Department of Preventive Medicine, Monash University, Department of Epidemiology and Preventive Medicine, Victoria, Australia

2Department of Medicine, Monash University, Southern Clinical School, Monash University, Clayton, Victoria, Australia

\section{Correspondence to}

Professor C A Brand, Centre of Research Excellence in Patient Safety (CREPS), Department of Preventive Medicine, Monash

University, Department of Epidemiology and Preventive

Medicine, Level 3

MacFarlane-Burnet Tower, 89 Commercial Road Melbourne, Victoria 3004, Australia; caroline.brand@med.monash. edu.au

Data sharing statement The full data-extraction algorithm can be obtained on request from VS (vijaya.sundararajan@med. monash.edu.au)

Accepted 4 March 2010 Published Online First 17 June 2010

\section{ABSTRACT}

Objectives To document the burden of in-hospital falls and fractures, and to identify factors that may increase the risk of these events.

Design A retrospective cohort analysis

Setting The study was set in the State of Victoria, Australia.

Participants Hospital episode data collected in the Victoria Admitted Episodes Dataset, for all multiday-stay patients 18 years or more admitted to Victorian public hospitals; 1 July 1998 to 30 June 2008. Diagnoses were defined by the International Classification of Disease, 10th Revision, Australian Modification (ICD-10-AM), which includes an in-hospital diagnostic timing code. Outcome measures included rates of in-hospital falls and fractures, length of hospital stay and mortality. Variables included in risk adjustment included financial year, individual demographic and comorbidity data, and hospital characteristics.

Results There were 3345415 episodes: 21250 (0.64\%) in-hospital falls and 4559 (0.14\%) fractures. In-hospital fall (IHF) episode rates increased over the study period, but fracture episode rates were stable. Mortality (HR 1.3, $\mathrm{Cl} 1.3$ to 1.5 ) and length of stay (median 19 days vs 5 days, $p<0.0001)$ were increased with IHF. Risk factors for IHF included dementia (rate ratio $1.7, \mathrm{Cl} 1.6$ to 1.8) and delirium (rate ratio $1.8, \mathrm{Cl} 1.6$ to 2.0).

Conclusions Routinely collected data that include a hospital diagnostic timing code offer a standard method of quantifying in-hospital falls and fractures. Unselected in-hospital falls data may be subject to reporting and documentation bias. The utility of using robust selected injuries such as IHF-related fracture as a quality-of-care indicator requires further investigation.

\section{INTRODUCTION}

International studies indicate that $3.2-10.6 \%$ of hospitalisations are associated with an adverse event, with a substantial impact on patient health outcomes and health costs. ${ }^{1-3}$ Falls are serious adverse events associated with increased length of in-hospital stay, functional decline $e^{4-7}$ and litigation claims. $^{8}$

Accurate measurement of adverse events is important for understanding and reporting the performance of healthcare systems. For in hospital falls (IHF), there is a wide variation in the reported burden, reflecting complexity of the problem, heterogeneity in study design, patient population, fall case-definition ${ }^{9}$ and contextual factors. Similarly, there is conflicting information about risk factors for IHF and fall-related injuries. Understanding these factors is important, not only to inform interventional strategies, but also for development of robust risk adjustment models for comparative benchmarking of organisational performance.

The main data source for IHF are incident reporting systems. These provide important qualitative information about the nature and causation of falls ${ }^{10}$ and safety culture, ${ }^{11}$ but do not provide robust epidemiological information for monitoring system performance. ${ }^{12} 13$ Medical record review and prospective observational studies provide more detailed clinical information about potential risk factors but are time-consuming and costly to conduct. $^{1} 14 \quad 15$ Routinely collected data are an alternative source of information about IHF events that capture data for the whole population. To date, the utility of monitoring IHF using routinely collected data has not been widely researched with existing studies limited by the absence of timing codes that accurately identify in-hospital events. ${ }^{16} 17$

The purpose of this study was to perform a retrospective population cohort study to measure rates of public hospital IHF and fall-related fractures (FRFx) and to identify factors which may increase the risk of these two events using routinely collected hospital discharge data over a 10-year period from 1 July 1998 to 30 June 2008.

\section{METHODS}

This study was approved by Monash University, Standing Committee on Ethics in Research Involving Humans.

\section{Study setting and population}

All residents in Australia have free access to publicly funded hospitals and may also choose to have private insurance for care in private hospitals. More than 5 million people reside in Victoria, the second largest State in Australia. ${ }^{18}$ The analysis sample included all public hospital discharge episodes in the State of Victoria included within the Victorian Admitted Episodes Dataset (VAED) for persons aged 18 years or more, admitted for 2 days or more between 1 July 1998 and 30 June 2008

The VAED was developed to meet national reporting obligations and support output-based funding of Victorian hospitals. ${ }^{19}$ It includes demographic, administrative and clinical information, and is externally linked to the Victorian Death Registrations. Since 1998, clinical information in the VAED has been coded using the International Classification of Diseases, 10 Revision, Australian Modification (ICD-10-AM). There are now 40 diagnostic code fields. For each diagnostic code, the 
timing of diagnosis is indicated by a corresponding variable which can take on several values: $\mathrm{P}$ (principal reason for admission), A (associated condition-comorbidity) or C (a new diagnosis arising during admission). ${ }^{20} 21$ Good to excellent coding quality of ICD-10-AM has been demonstrated for principal procedure codes, many diagnostic codes and coding of comorbid diagnoses. ${ }^{22}$

An IHF was defined as a fall event that occurred at any time during a single multiday stay hospital episode and was coded for inclusion in the VAED. A FRFx was defined as any fracture that occurred during a single hospital episode in which there was also documented an IHF and which was coded within the VAED. ICD-10-AM codes were used to define falls (W codes), injuries ( $S$ codes) and fractures (T codes) (table 1). Coders follow standard coding criteria for the inclusion of additional diagnoses; therefore, only IHF associated with analgesia administration, radiological investigation, increased clinical monitoring or injuries are coded. Fractures without falls were excluded, as these may represent misclassification of prehospital fractures diagnosed late or pathological fracture.

In the most recent audit of coding quality in Victoria, conducted in 2005-2006 of 10010 (approximately 1\% of all non-chemotherapy/non-dialysis) public hospital discharges, the prevalence of in-hospital falls based on the coding algorithm above was $3.64 \%$ in the original hospital coding versus $3.60 \%$ for the auditors; furthermore, using the auditor's codes as the reference standard, the sensitivity was $95.3 \%$ and the specificity 99.8\%. The $\kappa$ was 0.94 (personal communication).
Further elements of the data for analysis are summarised in table 1 . The Charlson condition diagnoses and additional fall and fracture risk conditions (osteoporosis, stroke, Parkinson's disease, ataxia, visual impairment, deafness, delirium) were included in risk adjustment; these comorbidities included only those present on admission. In-hospital comorbid diagnoses, flagged by the C-diagnosis timing code, were excluded. ${ }^{23} 24$

\section{Statistical analysis}

The data were extracted and analysed using SAS8.2 (SAS Institute, Cary, North Carolina). ${ }^{25}$ Crude rates of IHF were expressed as rate/1000 bed days. A descriptive analysis was undertaken to investigate associations between covariates of interest and fall events.

Multivariable Poisson regression models, corrected for overdispersion (PROC GENMOD), were then fitted to ascertain independent associations for experiencing an IHF or FRFx. The risk of FRFx was considered in two ways; for the whole population on admission (FRX model 1, denominator all episodes) and for those who experienced an IHF (FRFx model 2, denominator IHF episodes).

To assess the impact of fall-related events on survival, an index admission was defined for patients with a first IHF after 1 July 2002 (no IHF or presenting fall between 1 July 1998 and 30 June 2002). Controls were matched to these cases by age and gender from a randomly selected sample population without IHF with a first public hospital admission after 1 July 2000. Survival was defined from the date of the index admission to the date of death

Table 1 Summary of data-extraction variables

\begin{tabular}{|c|c|}
\hline & Covariate definition/label \\
\hline Fall & ICD-10-AM* three-digit codes W01, W03, W04, W05, W06, W07, W08, W10, W13, W17, W18, W19 \\
\hline Injury & Two-digit codes T0, T1, T8, T9 and Three-digit code T79 \\
\hline Fracture & Three-digit codes S12, S22, S32, S42, S52, S62, S72, S82, S92,T02, T08, T10, T12 \\
\hline Event timing & Diagnosis timing code of ' $\mathrm{C}$, ' indicating that diagnosis occurred in hospital \\
\hline Length of stay & Days \\
\hline Year of fall & Financial year (1 July to 30 June) \\
\hline Age & 5-year groups \\
\hline Gender & Male, female \\
\hline Marital status & Yes/no \\
\hline Australian-born & Yes/no \\
\hline English-speaking country of birth & Yes/no \\
\hline Geographic setting & Metropolitan/rural \\
\hline Socio-economic $\dagger$ & $\begin{array}{l}\text { Based on the geographic area of residence, the lowest quartile of Socio-Economic Indexes for Areas } \\
\text { score versus top three quartiles is presented for education occupation and education resources }\end{array}$ \\
\hline Hospital care type & Acute/newborn, palliative, geriatric, mental/alcohol-related, rehabilitation, interim, nursing home \\
\hline Admission type & Emergency, elective \\
\hline Admission source & Home, transfer, other \\
\hline Stay type & Sameday, multiday \\
\hline Hospital type & Teaching, other \\
\hline Diagnostic-related group & Medical, surgical, other \\
\hline Previous fall history & Documentation in previous separation as presenting or in hospital fall (yes, no) \\
\hline Presenting with fall & $P$ fall (yes, no) \\
\hline \multicolumn{2}{|c|}{ Comorbidities other than those listed in the Charlson Index } \\
\hline Ataxia & Four-digit codes G110, G111, G112, G113, G114, G118, G119, R270, R278 \\
\hline Deafness & Three-digit code H90, four-digit codes; H910, H911, H912, H913, H918, H919, 0780 \\
\hline Delirium & $\begin{array}{l}\text { Four-digit codes F050, F051, F058, F059, F104, F106, F114, F124, F134, F144, F154, F164, F174, F184, } \\
\text { F194, F430 }\end{array}$ \\
\hline Neuromyalgia & Four-digit code M792 \\
\hline Osteoporosis & Three-digit codes; M80, M81, M82 \\
\hline Parkinson's disease & Three-digit code G20 \\
\hline Vision impairment & Three-digit codes; H53, H54 \\
\hline
\end{tabular}


or the end of follow-up (30 June 2008). The risk of mortality was investigated using a Cox proportional hazards model, adjusted for covariates of interest and expressed as a HR. The FRF Cox proportional hazards model was conducted among the cases of IHF in this truncated sample. The relationships between IHF, FRFx and median length of stay were assessed using the nonparametric Wilcoxon Rank Sum test.

\section{RESULTS}

The dataset included 3345415 episodes, of which 21250 $(0.64 \%)$ were coded with an IHF, and 4559 were coded with an FRFx (17.6\% IHF and 0.14\% total episodes). Notably, 827 (18\%) in hospital fractures) occurred in an episode without a coded IHF; in which there was an associated diagnosis of cancer in 71 $(8.6 \%)$ or metastatic cancer (3.8\%). There were 1656 in hospital hip fractures (44.4\% all in hospital fractures). Overall FRFx accounted for $18.5 \%$ of all in-hospital injuries.

IHF were more common when the presenting episode diagnosis was a fall $(1.5 \%$ vs $0.6 \%, p<0.0001)$ or an injury $(1.0 \%$ vs $0.6 \%, \mathrm{p}<0.0001)$. Similarly FRFx was more prevalent in those admitted with a fall $(21.2 \%$ vs $17.1 \%, \mathrm{p}<0.0001)$, any fracture $(23.8 \%$ vs $16.8 \%, p<0.0001)$ or hip fracture $(25.8 \%$ vs $17 \%$, $\mathrm{p}<0.0001$ ). Of those admitted with hip fracture, $8.7 \%$ experienced a further FRFx.

The general characteristics of all hospital discharges are summarised in table 2. Nearly one-third of all episodes occurred in rural settings. Over $50 \%$ of episodes were for individuals over 50 years of age. The proportion over 80 years $(19.4 \%$ total population) was higher for those with an IHF (50.9\%) and FRFx (57.3\%). There was a higher proportion of women $(63 \%)$ in the

Table 2 Summary of demographic and clinical details for hospital episodes associated with falls and fall-related fractures (FRF) in Victorian public hospitals between 1998 and 2008

\begin{tabular}{|c|c|c|c|c|c|c|c|c|}
\hline & \multicolumn{2}{|c|}{ All episodes $\mathrm{N}=3344588$} & \multicolumn{2}{|c|}{$\begin{array}{l}\text { Episodes for IHF } \\
N=21250\end{array}$} & \multirow[b]{2}{*}{ Row (\%) } & \multicolumn{2}{|c|}{$\begin{array}{l}\text { Episodes for FRFx } \\
N=3732\end{array}$} & \multirow[b]{2}{*}{ Row (IHF) \% } \\
\hline & $\mathbf{N}$ & Percentage & $\mathbf{N}$ & Percentage & & $\mathbf{N}$ & Percentage & \\
\hline \multicolumn{9}{|l|}{ Age (years) } \\
\hline $18-39$ & 945222 & 28.3 & 679 & 3.2 & 0.1 & 56 & 1.5 & $8.2 \dagger$ \\
\hline $40-49$ & 302701 & 9.1 & 650 & 3.1 & 0.2 & 63 & 1.7 & $9.7 \dagger$ \\
\hline $50-59$ & 343593 & 10.3 & 1035 & 4.9 & 0.3 & 140 & 3.8 & 13.5 \\
\hline $60-69$ & 451678 & 13.5 & 2129 & 10.0 & 0.5 & 302 & 8.1 & $14.2^{*}, \dagger$ \\
\hline $70-79$ & 654272 & 19.6 & 5942 & 28.0 & 0.9 & 1034 & 27.7 & 17.4 \\
\hline $80-89$ & 524673 & 15.7 & 8179 & 38.5 & 1.6 & 1638 & 43.9 & $20.0+$ \\
\hline $90+$ & 123276 & 3.7 & 2636 & 12.4 & 2.1 & 499 & 13.4 & 18.9 \\
\hline \multicolumn{9}{|l|}{ Gender } \\
\hline Female & 1952479 & 58.4 & 11020 & 51.9 & 0.6 & 2350 & 63.0 & $21.3^{*}$ \\
\hline Male & 1392936 & 41.6 & 10230 & 48.1 & $0.7^{*}$ & 1382 & 37.0 & $13.5 \dagger$ \\
\hline Australian-born & 2246610 & 77.2 & 13485 & 63.5 & $0.6 \dagger$ & 2387 & 64.0 & 17.7 \\
\hline English-speaking country of birth & 2535841 & 75.8 & 15724 & 74.0 & $0.6 \dagger$ & 2768 & 74.2 & 17.6 \\
\hline Married & 1640294 & 49.8 & 8481 & 39.9 & $0.5 \dagger$ & 1282 & 34.3 & $15.1 \dagger$ \\
\hline Rural & 942013 & 28.9 & 4894 & 23.3 & $0.5 \dagger$ & 817 & 22.1 & 16.7 \\
\hline \multicolumn{9}{|l|}{ Socio-economic } \\
\hline Education occupation & 928424 & 27.8 & 5422 & 25.5 & $0.6 \dagger$ & 918 & 24.6 & 16.9 \\
\hline Education resources & 473998 & 14.2 & 2846 & 13.3 & 0.6 & 465 & 12.5 & 16.3 \\
\hline \multicolumn{9}{|l|}{ Comorbid conditions } \\
\hline Cancer & 328146 & 9.8 & 2876 & 13.5 & $0.9^{*}$ & 405 & 10.9 & $14.1^{*}$ \\
\hline Metastatic cancer & 161561 & 4.8 & 1557 & 7.3 & $1.0^{*}$ & 217 & 5.8 & $13.9^{*}$ \\
\hline Chronic heart failure & 216452 & 6.5 & 2589 & 12.2 & $1.2^{*}$ & 483 & 12.9 & 18.7 \\
\hline Cerebrovascular disease & 158876 & 4.8 & 2617 & 12.3 & $1.7^{*}$ & 431 & 11.6 & 16.5 \\
\hline Connective tissue disease & 25452 & 0.8 & 223 & 1.1 & $0.9^{*}$ & 57 & 1.5 & 25.6 \\
\hline Chronic pulmonary disease & 243823 & 7.3 & 1838 & 8.7 & $0.8^{*}$ & 359 & 9.6 & 19.5 \\
\hline Dementia & 151519 & 4.5 & 4017 & 18.9 & $2.7^{*}$ & 844 & 22.6 & $21.0^{*}$ \\
\hline Diabetes & 246011 & 7.4 & 1896 & 8.9 & $0.8^{*}$ & 363 & 9.7 & 19.2 \\
\hline Severe diabetes & 102253 & 3.1 & 1301 & 6.1 & $1.3^{*}$ & 177 & 4.7 & 13.6 \\
\hline HIV & 6753 & 0.2 & 57 & 0.3 & 0.8 & 9 & 0.2 & 15.8 \\
\hline Liver disease & 16910 & 0.5 & 212 & 1 & $1.3^{*}$ & 34 & 0.9 & 15.7 \\
\hline Severe liver disease & 12810 & 0.4 & 151 & 0.7 & $1.2^{*}$ & 20 & 0.5 & $13.3^{*}$ \\
\hline Myocardial infarction & 113555 & 3.4 & 742 & 3.5 & 0.7 & 122 & 3.3 & 16.4 \\
\hline Paraplegia & 88510 & 2.7 & 1635 & 7.7 & $1.9^{*}$ & 247 & 6.6 & 15.1 \\
\hline Peptic ulcer disease & 23912 & 0.7 & 179 & 0.8 & 0.8 & 33 & 0.9 & 15.6 \\
\hline Peripheral vascular disease & 36571 & 1.1 & 343 & 1.6 & $0.9^{*}$ & 62 & 1.7 & 18.1 \\
\hline Renal disease & 164412 & 4.9 & 2203 & 10.4 & $1.3^{*}$ & 347 & 9.3 & 15.8 \\
\hline Ataxia & 9382 & 0.3 & 205 & 1.0 & $2.2^{*}$ & 26 & 0.7 & 12.7 \\
\hline Delirium & 45092 & 1.4 & 1272 & 6.0 & $2.8^{*}$ & 240 & 6.4 & 18.9 \\
\hline Hearing deficit & 16459 & 0.5 & 417 & 2.0 & $2.5^{*}$ & 69 & 1.9 & 16.6 \\
\hline Osteoporosis & 34521 & 1.0 & 663 & 3.1 & $1.9^{*}$ & 228 & 6.1 & $34.4^{*}$ \\
\hline Parkinson's disease & 30819 & 0.9 & 933 & 4.4 & $3.0^{*}$ & 170 & 4.6 & 18.2 \\
\hline Visual deficit & 27934 & 0.8 & 540 & 2.5 & $1.9^{*}$ & 97 & 2.6 & 18.0 \\
\hline
\end{tabular}

*Higher prevalence of in-hospital falls (IHF) or FRFx.

†Lower prevalence of IHF or FRFx associated with variable, assessed using univariate statistical analysis, $\mathrm{p} \leq 0.001$. 
FRFx group. The most prevalent comorbidities among the total population were: cancer, diabetes, pulmonary disease and chronic heart failure. However, dementia was the major comorbid condition associated with IHF (18.9\%) and FRFx $(22.6 \%)$. There was also a higher proportion of episodes with a comorbid diagnosis of delirium among IHF (6.0\%) and FRFx (6.4\%) compared with the total population (1.4\%).

Hospital characteristics are summarised in table 3. Emergency admissions accounted for fewer FRFx episodes (44.5\% vs $52.4 \%$ total). Acute and newborn care type accounted for the highest volume of episodes for all groups, but geriatric type was prominent in IHF (14.4\%) and FRFx (20.3\%) compared with total (2.8\%), as was rehabilitation type (IHF 10.3\%, FRFx 14.2\%, total $3.8 \%)$.

Unadjusted rates of IHF increased over the 10-year period (from 0.41 to $0.88 \mathrm{IHF} / 1000$ bed days) (table 4). The fracture rates were discordant between models. Rates for FRFx (0.11 to $0.13 / 1000$ bed days) remained stable in Model 1 but were markedly reduced in FRFx Model 2 (0.09 to 0.03/1000 bed days). These trends were also noted in the adjusted data (figure 1).

In the Poisson regression model (table 5), the strongest associations with IHF were: increasing age and comorbidities; HIV (RR 2.2, CI 1.4 to 3.5), liver disease (RR 1.8, CI 1.4 to 2.4), Parkinson's disease (RR 1.7, CI 1.5 to 1.9), ataxia (RR 1.6, CI 1.2 to 2.0), dementia (RR 1.7, CI 1.6 to 1.8) and delirium (RR 1.8, CI 1.6 to 2.0 ). In FRFx Model 2 there was similarly a strong association with increasing age, and comorbidities but an inverse relationship with male gender (RR 0.8 , CI 0.7 to 0.9 ). Of interest, a history of previous fall was associated with a somewhat lower IHF rate (RR 0.8, CI 0.8 to 0.9) and FRFx (model 1) rate (RR 0.8, CI 0.7 to 0.9), but this was not the case for FRFxmodel 2 . There were no additional factors in this second fracture model for which an independent association with fracture could be identified.

The median length of stay (LOS) was longer for episodes associated with an IHF (19 days vs 5 days, $\mathrm{p}<0.0001$ ), and this difference was greater for episodes associated with FRFx (23 days vs 5 days, $\mathrm{p}<0.0001$ ).
The HR for mortality for an IHF was increased at 1.3 (CI 1.3 to $1.5, \mathrm{p}<0.0001)$. An FRFx conferred no additional mortality risk (HR 1.1, CI 1.0 to 1.3 ).

\section{DISCUSSION}

We have quantified the burden of IHF and FRFx across a broad range of public hospitals in Victoria, Australia. While the definition of falls in this study was limited by the Australian coding standard to more serious falls and fall-related events, the dataextraction algorithm provides a reproducible process for data analysis. It may be suitable for benchmarking comparisons where ICD-10 coding forms the basis of data definition, and comparable quality of the coding can be established. With the specific in-hospital diagnosis timing codes, we have been able to provide population level figures about falls in all hospital episode types. In contrast, the Agency for Healthcare Research and Quality (AHRO) in the USA has included within their Patient Safety Indicator set a falls indicator in which the population of interest is restricted to post surgical falls in an attempt to avoid including false-positive adverse events. The introduction of the 'present on admission' flag throughout the US and the 'condition-onset flag' Australia-wide in late 2008 may widen the utility of standardised algorithms such as those presented here.

There is limited ability to compare our results to previous studies due to differences in methodology. Our baseline IHF rate is significantly lower than that reported in other Australian studies based on incident reporting data (5.4-7.9 falls/1000 bed days). ${ }^{26} 27$ Although rates of IHF within different (often small) patient populations and settings have been reported to vary between 0.3 and $19.0 / 1000$ bed days based on incident reporting data, ${ }^{28}$ our low rate almost certainly reflects the definition of the denominator IHF for this study and the failure therefore to capture all IHF, especially falls of lower severity. It is also difficult to compare fall-related injury rates. ${ }^{9}$ Overall, previous studies based on a variety of settings and patient populations report the proportion of fall injuries to be between $26.7 \%$ and $70 \%$ of all fall incidents, and the proportion of fracture injuries to vary between 2.1 and $6 \% .^{727-30}$ Our higher proportion of fracture injuries is

Table 3 Summary of hospital characteristics for in-hospital falls (IHF) and fall-related fractures (FRFx) between 1 July 1998 and 30 June 2008

\begin{tabular}{|c|c|c|c|c|c|c|c|c|}
\hline Characteristic & \multicolumn{2}{|c|}{$\begin{array}{l}\text { Total episodes } \\
\mathrm{N}=3344588\end{array}$} & \multicolumn{2}{|c|}{$\begin{array}{l}\text { IHF episodes } \\
\mathrm{N}=\mathbf{2 1 2 5 0}\end{array}$} & Row percentage & \multicolumn{2}{|c|}{$\begin{array}{l}\text { FRFx episodes } \\
\mathrm{N}=3732\end{array}$} & Row percentage (of IHF \\
\hline Emergency admission & 1751778 & 52.4 & 11397 & 53.6 & 0.7 & 1659 & 44.5 & $14.6 \dagger$ \\
\hline Acute and newborn $\ddagger$ & 2913988 & 87.1 & 13787 & 64.9 & 0.50 & 1953 & 52.3 & $14.2 \dagger$ \\
\hline Palliative & 39931 & 1.2 & 647 & 3.0 & 1.6 & 123 & 3.3 & 19.0 \\
\hline Geriatric & 93542 & 2.8 & 3061 & 14.4 & 3.3 & 759 & 20.3 & $24.8^{*}$ \\
\hline Interim care§ & 9865 & 0.3 & 169 & 0.8 & 1.7 & 24 & 0.6 & 14.2 \\
\hline Interim care-nursing§ home type & 14799 & 0.4 & 614 & 2.9 & 4.2 & 127 & 3.4 & 20.7 \\
\hline \multicolumn{9}{|l|}{ Admission source } \\
\hline Home & 1451945 & 43.4 & 7741 & 36.4 & 0.5 & 996 & 26.7 & $12.9 \dagger$ \\
\hline Transfer & 346474 & 10.4 & 5216 & 24.1 & $1.5^{*}$ & 1101 & 29.5 & $21.5^{*}$ \\
\hline
\end{tabular}

*Higher prevalence of IHF or FRFX.

†Lower prevalence of IHF or FRFx associated with variable, assessed using uni-variate statistical analysis, $\mathrm{p} \leq 0.001$.

$\ddagger$ Acute and Newborn care type includes all acute hospital admissions other than those specified. For this analysis, only persons 18 years or more are included in the analysis.

$\S$ There are two types of interim care; those where persons have already been designed nursing home type ( 35 days of continuous care, assessed by aged care assessment service) and those without such a designation.

TEither a previous presenting fall or an in-hospital fall hospital episode.

$\mathrm{EOH}$, alcohol related conditions. 
Table 4 Unadjusted rates of in-hospital fall (IHF) and fall-related fracture (FRF) separations between 1 July 1998 and 30 June 2008

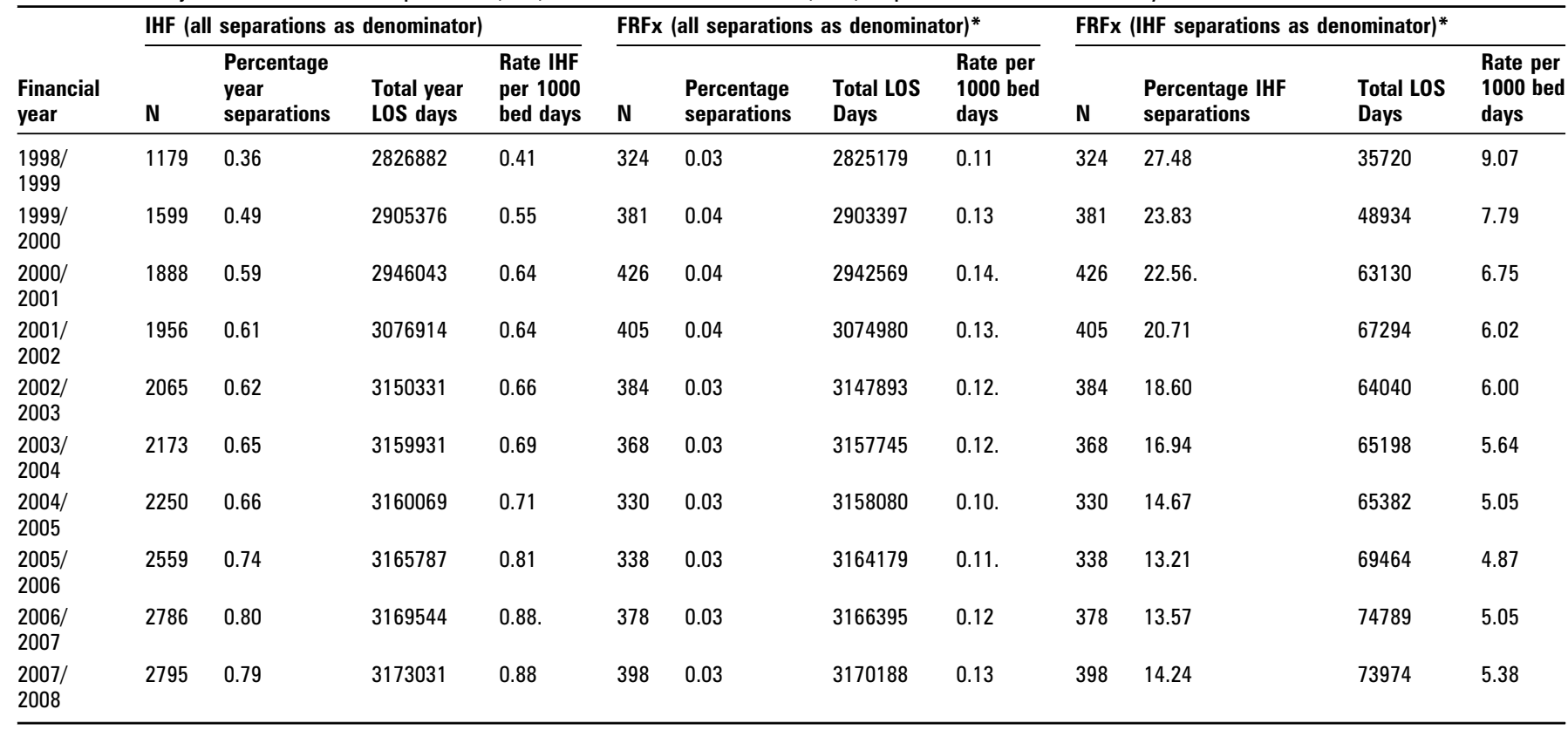

*The denominator does not include episodes in which there was an in hospital fracture but no associated fall.

LOS, length of stay.

again probably due to application of the Australian coding standard and therefore differences in injury definition which reduce the IHF denominator to include only more serious falls.

The results highlight the need for caution in interpreting trends and in choosing useful indicators of quality of care. Ideally, a useful indicator can identify systemic variation after adequate adjustment for patient case mix. Increasing adjusted IHF rates may reflect changes in coding practice associated with increasing awareness, better reporting of fall events in hospital and consequently reporting of less serious events as well as real changes in IHF rates. The differences in trends noted for adjusted IHF rates and fracture rates support the premise that the IHF rates reported in the later years of our analysis may be of lower severity: the stable fracture rates in model 1 and decreasing FRFx rates in Model 2 support a dilution effect due to more reporting of less serious falls. Therefore, FRFx rates are likely to be a more useful indicator of system performance, although, in view of relatively low event rates, further investigation is needed to determine the utility of such an indicator for interorganisational comparisons.
In addition to monitoring high-level indicators of system performance, there is increasing interest in measuring individual organisational performance, benchmarking and reporting performance to internal and external stakeholders, including the public. Such comparative data require robust risk adjustment models to ensure credible figures to drive quality improvement at a local level and to avoid time-consuming investigation of false-positive events. The purpose of our study was not to develop and validate a fall or fracture risk prediction tool but to investigate the utility of routinely collected data for contributing to understanding individual and population risk, in particular for identifying factors for inclusion in future riskadjustment models. In so doing, we have confirmed a number of previously reported important IHF factors. ${ }^{31}$ The relationship between structural care type other than acute and newborn for FRFx rates is expected. ${ }^{32}$ Of particular importance is the strong association with cognitive impairment (dementia and delirium) which will become an increasing challenge to organisations treating an ageing population. The association of HIV with IHF and FRFx is in keeping with known increased prevalence of
Figure 1 Burden of IHF and FRFx between 1st July 1998 and 30th June 2008. Rate ratios adjusted for variables of interest.

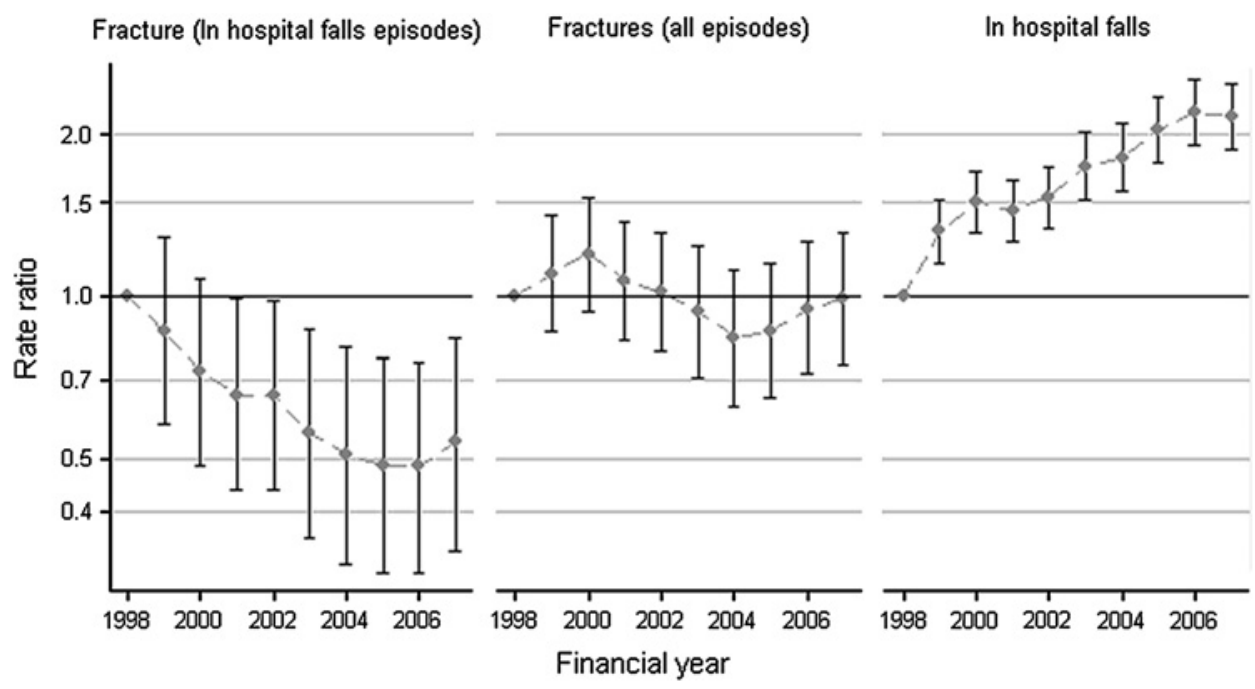


Table 5 Poisson regression model: summary of the rate ratios for covariates associated with in-hospital falls and fractures for Victorian public hospital episodes between 1 July 1998 and 30 June 2008, adjusted for variables of interest

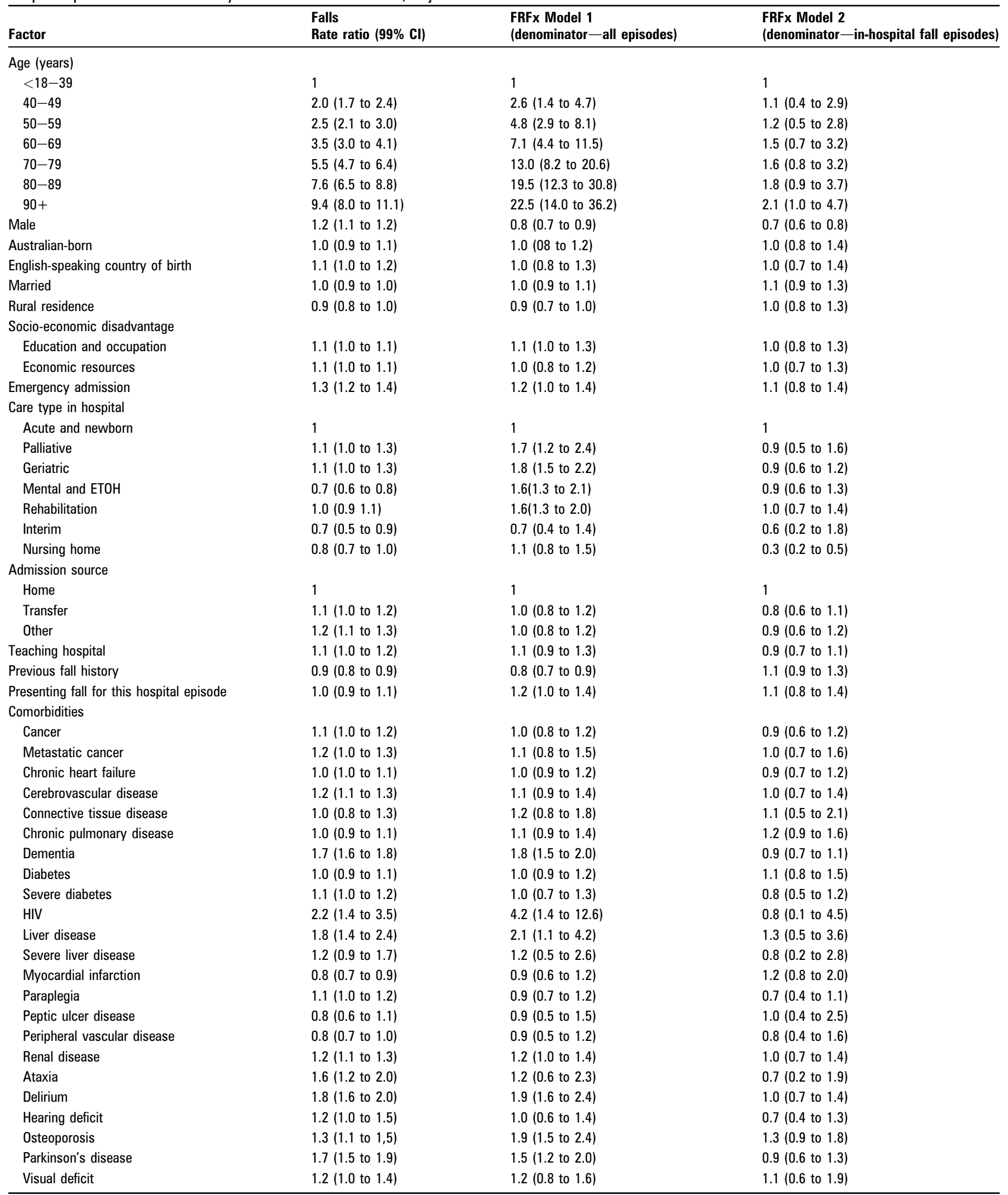

EOH, alcohol related conditions; FRFx, fall-related fracture.

osteopenia and osteoporosis and recent evidence for higher fracture prevalence compared with control populations. ${ }^{33}$

We acknowledge the well-reported limitations of using routinely collected hospital data whose primary purpose is for case mix funding rather than clinical outcomes monitoring. ${ }^{34}$ In addition to coding errors, ascertainment of adverse events has been shown to be related to the completeness of record documentation. ${ }^{1}$ Our study reinforces the limitation of using such data 
for understanding associations without additional contextual information, particularly where there is statistical significance but relatively weak increased risk, for instance for male gender on falls risk. Overall, these data are hypothesis-generating and must be supported by more focused observational data to be useful at an organisational level for quality-improvement purposes.

Of interest, presentation with or previous history of a fallrelated hospital episode was associated with lower rate ratios of IHF, suggesting there may have been greater awareness and implementation of preventive strategies. Alternatively, patients may have been immobilised by the injury that led to admission, although this association was not found in the fracture models. Similarly, there was no association between clinical comorbidity data and fractures in fracture model 2. We may have failed to show significant associations with fracture risk and mortality outcomes due to small overall fracture numbers; however, additional factors, not currently measured, may be influencing fracture risk in those who fall. ${ }^{35}$ We have not differentiated risk for single versus multiple falls. ${ }^{36}$ These will not be captured within the discharge dataset unless there were different injurious conditions - for example, a laceration on one occasion and a fracture on another. Finally, our regression models included both individual and aggregate risk factors, and we may not have accounted for all cluster effects.

In Australia, it is estimated that the total estimated health cost attributable to fall injuries will increase threefold between 2001 and 2051 , rising to $\mathrm{A} \$ 1375$ million per year. ${ }^{37}$ Our analysis illustrates the strengths and weakness of alternate methods to quantifying this burden and contributes to efforts to develop a monitoring system to follow the impact of interventions to reduce hospital associated fall-related injuries in high-risk populations.

Acknowledgements The authors would like to thank A Gorelik, for her contribution to defining the falls data-extraction algorithm, J Shepheard, for advice regarding ICD-10-AM coding, K Hill and VK Srikanth, for kindly reading and providing advice on the paper.

Competing interests VS is a senior medical advisor to the Department of Health, Victoria.

Ethics approval Ethics approval was provided by Monash University, Standing Committee on Ethics in Research Involving Humans.

Contributors $\mathrm{CAB}$ was responsible for the conception of the study and writing the draft paper. She contributed to the design of the study and interpretation of the analysis. VS was responsible for the design of the study, the statistical analysis and its interpretation, and contributed to writing of the paper. VS had full access to all of the data (including statistical reports and tables), and CAB had full access to summarised data analysis in the study and can take responsibility for the integrity of the data and the accuracy of the data analysis.

Provenance and peer review Not commissioned; externally peer reviewed.

\section{REFERENCES}

1. Wilson RM, Runciman WB, Gibberd RW, et al. The quality in Australian health care study. Med J Aust 1995;163:458-71.

2. Thomas EEJ, Studdert DDM, Runciman WWB, et al. A comparison of iatrogenic injury studies in Australia and the USA. I: context, methods, casemix, population, patient and hospital characteristics. Int J Qual Health Care 2000;12:371-8.

3. Brennan TA, Leape LL, Laird NM, et al. Incidence of adverse events and negligence in hospitalized patients. Results of the Harvard Medical Practice Study I [comment]. N Engl J Med 1991;324:370-6.

4. Kannus P. Preventing osteoporosis, falls, and fractures among elderly people. Promotion of lifelong physical activity is essential [comment]. BMJ. 1999;318:205-6.

5. Tinetti M. Preventing falls in elderly persons. N Engl J Med 2003;348:42-50.

6. Kannus P, Parkkari J, Niemi S, et al. Fall-induced deaths among elderly people. Am J Public Health (1971) 2005;95:422-4.
7. Nadkarni JB, lyengar KP, Dussa $\mathrm{C}$, et al. Orthopedic Injuries following falls by hospital in-patients. Gerontology 2005;51:329-33.

8. Oliver D, Killick S, Even T, et al. Do falls and falls-injuries in hospital indicate negligent care-and how big is the risk? a retrospective analysis of the NHS Litigation authority database of clinical negligence claims, resulting from falls in hospitals in England 1995 to 2006. Oual Saf Health Care 2008:17:431-6.

9. Hauer K, Lamb SE, Jorstad EC, et al. Systematic review of definitions and methods of measuring falls in randomised controlled fall prevention trials. Age \& Ageing 2006;35:5-10.

10. Healey F, Scobie S, Oliver D, et al. Falls in English and Welsh hospitals: a national observational study based on retrospective analysis of 12 months of patient safety incident reports. Qual Saf Health Care 2008;17:424-30.

11. Hutchinson A, Young TA, Cooper $\mathrm{KL}$, et al. Trends in healthcare incident reporting and relationship to safety and quality data in acute hospitals: results from the national reporting and learning system. Qual Saf Health Care 2009 18:5-10.

12. Sari AB-A, Sheldon TA, Cracknell A, et al. Sensitivity of routine system for reporting patient safety incidents in an NHS hospital: retrospective patient case note review. BMJ (Clin Res Ed). 2007;334:79.

13. Shorr RI, Mion LC, Chandler AM, et al. Improving the capture of fall events in hospitals: combining a service for evaluating inpatient falls with an incident report system. J Am Geriatr Soc 2008;56:701-4.

14. Murff HJ, Patel VL, Hripcsak G, et al. Detecting adverse events for patient safety research: a review of current methodologies. J Biomed Inform 2003; $\mathbf{3 6}: 131-43$

15. Thomas EJ, Petersen LA, et al. Measuring errors and adverse events in health care. $J$ Gen Intern Med 2003;18:61-7.

16. Bates D, Cullen DJ, Laird N, et al. Incidence of adverse drug events and potentia adverse drug events: implication for prevention. JAMA 1995;274:29-34.

17. Iezzoni L, Daley J, Heeren T, et al. Identifying complications of care using administrative data. Med Care 1994:32:700-15.

18. Australian Bureau of Statistics (ABS). Australian demographic statistics. 2008 http://www.abs.gov.au/ausstats (cited 11 Apr 2009).

19. The Victorian Admitted Episodes Dataset. User manual. 2002 http://health.vic. gov.au/hdss/vaed/index (accessed May 19 2009).

20. National Centre for Classification in Health (NCCH). Australian coding standards. 6th ed. Sydney, Australia: University of Sydney, 2008.

21. Jackson TT, Duckett SS, Shepheard JJ, et al. Measurement of adverse events using 'incidence flagged' diagnosis codes. J Health Serv Res Policy 2006;11: $21-6$

22. Henderson TT, Shepheard JJ, Sundararajan W. Quality of diagnosis and procedure coding in ICD-10 administrative data. Medical care 2006;44:1011-9.

23. Charlson ME, Pompei P, Ales KL, et al. A new method of classifying prognostic comorbidity in longitudinal studies: development and validation. J Chronic Dis 1987:40:373-83.

24. Sundararajan V, Henderson T, Perry C, et al. New ICD-10 version of the Charlson comorbidity index predicted in-hospital mortality. J Clin Epidemiol 2004; 57:1288-94.

25. SAS Institute Inc. SAS User's Guide. V.8. 2nd ed. Cary, NC, USA: SAS, 1999.

26. Hill $\mathbf{K}, \mathrm{Vu} \mathrm{M}$, Walsh $\mathrm{W}$. Falls in the acute hospital setting-impact on resource utilisation. Aust Health Rev 2007;31:471-7.

27. Brandis S. A collaborative occupational therapy and nursing approach to falls prevention in hospital inpatients. J Qual Clin Pract 1999:19:215-20.

28. Halfon P, Eggli Y, Van Melle G, et al. Risk of falls for hospitalized patients: A predictive model based on routinely available data. J Clin Epidemiol 2001:54:1258-66.

29. Enloe M, Wells TJ, Mahoney J, et al. Falls in acute care: an academic medica center six-year review. J Patient Saf 2005;1:208-14.

30. Hitcho EB, Krauss MJ, Birge $S$, et al. Characteristics and circumstances of falls in a hospital setting: a prospective analysis. J Gen Intern Med 2004;19:732-9.

31. Sadigh S, Reimers A, Andersson R, et al. Falls and fall-related injuries among the elderly: a survey of residential-care facilities in a Swedish municipality. J Community Health 2004;29:129-40.

32. Hignett $\mathbf{S}$, Masud T. A review of environmental hazards associated with in-patient falls. Ergonomics 2006;49:605-16.

33. Triant VA, Brown $\mathrm{TT}$, Lee $\mathrm{H}$, et al. Fracture prevalence among human immunodeficiency virus (HIV)-infected versus non-HIV-infected patients in a large US healthcare system. J Clin Endocrinol Metab 2008;93:3499-504.

34. Iezzoni L. Assessing quality using administrative data. Ann Intern Med. 1997;127:666-74

35. Vassallo M, Vignaraja $\mathrm{R}$, Sharma JC, et al. The relationship of falls to injury among hospital in-patients. Int J Clin Pract 2005;59:17-20.

36. Vassallo M, Sharma JC, Allen SC. Characteristics of single fallers and recurrent fallers among hospital in-patients. Gerontology 2002;48:147-50.

37. Australian Commission for Safety and Quality in Healthcare (ACSOHC) Preventing falls and harm from falls in older people: Best practice guidelines for Australian hospitals and residential aged care facilities. Canberra, Australia: Commonwealth of Australia, 2008:1-195. 